\title{
Efectos de una dieta mediterránea tradicional en niños con sobrepeso y obesidad tras un año de intervención
}

\author{
F. Calatayud Sáeza , B. Calatayud Moscoso del Pradob, JG. Gallego Fernández-Pachecoc
}

aPediatra. Clínica infantojuvenil La Palma. Ciudad Real. España.

${ }^{b}$ Nutricionista. Clínica infantojuvenil La Palma. Ciudad Real. España.

'Médico de familia. CS n. ${ }^{\circ}$ I. Ciudad Real. España.

Resumen

Introducción: la estrategia preventiva y terapéutica de la obesidad ofrece un escaso desarrollo en el sistema asistencial. No hay evidencia suficiente para determinar qué dieta es la más eficiente. El objetivo del estudio fue valorar el efecto de una intervención basada en una dieta mediterránea/tradicional.

Pacientes y métodos: se realizó en niños de 2 a 14 años con sobrepeso y obesidad a partir de un programa nutricional denominado "Aprendiendo a comer". Se tomaron medidas antropométricas y se utilizaron test de calidad nutricional. No hubo restricción calórica, no se promovió actividad deportiva ni hubo tratamiento cognitivo-conductual. Se promocionó en todo el ámbito familiar. Se dio prioridad a los alimentos naturales, perecederos y de temporada.

Resultados: noventa y ocho niños completaron el estudio. El 61,2\% presentó normopeso al finalizar la intervención. Dejaron de ser obesos y de tener sobrepeso el $73,1 \%$ y el $82,6 \%$, respectivamente. La pérdida de masa grasa fue del $18,7 \%$, la masa magra aumentó adecuadamente y el índice de calidad nutricional KidMed fue óptimo en el 95\% al finalizar la intervención.

Conclusiones: frente a las dietas hipocalóricas y de bajo índice glucémico, proponemos una dieta basada en la dieta mediterránea/tradicional y desde Atención Primaria. La disminución observada en el percentil del índice de masa corporal es clínicamente relevante, con un descenso importante de niños con sobrepeso y obesidad. El porcentaje de masa grasa corporal disminuyó de forma significativa. La dieta que proponemos es fácil de cumplimentar y, junto con el programa de educación nutricional, consideramos que es el pilar fundamental del estudio.

Palabras clave: Obesidad infantil. Sobrepeso infantil. Índice de masa corporal. Índice glucémico. Masa grasa. Intervención dietética. Atención Primaria.

Fernando Calatayud Sáez, altayud@gmail.com y fcalatayud@ono.com Los autores declaran no presentar conflictos de intereses en relación con la preparación y publicación de este artículo.

Datos parciales de este estudio se han presentado en forma de comunicación en el Congreso de la Sociedad Española de Pediatría Extrahospitalaria y Atención Primaria, celebrado en Murcia en octubre de 2010, y en el 60. ${ }^{\circ}$ Congreso Nacional de la Asociación Española de Pediatría, celebrado en Valladolid en junio de 2011

El estudio ha sido aprobado por los Comités de Ética e Investigación del Hospital General de Ciudad Real, y se ha realizado con el consentimiento informado de padres y tutores, tal como se refleja en el apartado de métodos del manuscrito. 
Effects of the mediterranean traditional diet in overweight and obese children after one year of intervention

Abstract

Introduction: the preventive and therapeutic strategy for obesity offers a poor development in the health care system. There is not enough evidence to determine what diet is the most efficient. The purpose of the study was to evaluate the effect of an intervention based on a Traditional Mediterranean diet.

Methods: the study was conducted in overweight and obese children aged 2 to 14 years through the nutrition education program "Learning to eat". Anthropometric measurements and Mediterranean diet tests were used. There was neither calorie restriction nor physical activity promotion nor was cognitive-behavioral therapy. Mediterranean diet was promoted throughout the family environment. Priority was given to natural, perishable and seasonal foods.

Results: the study was completed by 98 children; $61.2 \%$ showed a normal weight at the end of the intervention. At the end of the study, $73.1 \%$ and $82.6 \%$ were no longer obese or overweight. The proportion of fat mass decreased $18.66 \%$, lean mass increased properly, and KidMed diet quality index was optimal in $95 \%$ after the intervention.

Conclusions: opposed to low-calorie and low glycemic index diets, we propose a diet based on the Mediterranean Traditional Diet and from Primary Care. The observed decrease in Body Mass Index is clinically relevant, with a significant decrease in the proportion of overweight and obesity. The percentage of body fat mass decreased significantly. The Traditional Mediterranean Diet is easy to complete and along with the nutritional education program proposed, we consider it as the main pillar of the study.

Key words: Childhood obesity. Childhood overweight. Body mass index. Glycemic index. Body fat mass. Dietary intervention. Primary care.

\section{Introducción}

El sobrepeso (SP) y la obesidad (OB) infantojuvenil han ido aumentando de manera exponencial en los últimos años, alcanzando en algunas comunidades occidentales hasta un tercio de su población y a más de la mitad de sus individuos adultos. Esto ha llevado a la Organización Mundial de la Salud a declararla como la epidemia más importante del siglo XXI"

Se considera que los cambios acaecidos en el estilo de vida, cada vez más sedentario, junto con la existencia de un balance energético positivo, prolongado a lo largo del tiempo, conducen a un depósito graso elevado en el organismo y son los determinantes clave en el desarrollo de la obesidad ${ }^{2,3}$.

Los niños y niñas con $O B$ tienden a perpetuarla en la adolescencia y a desarrollarla y afianzarla en la edad adulta ${ }^{4}$, con el aumento consiguiente de enfermedades crónicas como diabetes e hipertensión arterial y el incremento secundario del riesgo de enfermedad coronaria ${ }^{5} y$ de mortalidad 6 . Por otro lado, la obesidad se relaciona con alteraciones psico- 
patológicas como baja autoestima y depresión, condicionando un deterioro de la calidad de vida ${ }^{7}$. Todo ello, junto con las repercusiones económicas que ocasiona, la convierte en un problema de salud pública de primera magnitud.

A pesar de todo, la estrategia preventiva y terapéutica de la obesidad ofrece un escaso desarrollo en el sistema asistencial. La falta de consenso y la dificultad que supone la intervención sobre cambios de conducta en los estilos de vida conducen a menudo a resultados decepcionantes que llevan al desánimo a pacientes y profesionales ${ }^{8}$. Por otro lado, la mayoría de los estudios sobre el tratamiento de la obesidad realizan intervenciones sobre diferentes factores como dieta, ejercicio físico y terapia cognitivoconductual, lo que dificulta la evaluación del papel de la dieta, y los que se centran en el tratamiento nutricional no han aportado evidencia suficiente sobre qué dieta es la más efectiva ${ }^{9-13}$.

Aunque en España suele recomendarse la alimentación basada en la dieta mediterránea como la más adecuada para la población en general14, hay pocos estudios que evalúen su aplicación en los pacientes obesos ${ }^{15}$.

El objetivo del estudio fue valorar el efecto de una intervención basada en la aplicación de una dieta mediterránea/ tradicional (DM/T) y de un programa de educación nutricional ("Aprendiendo a comer"), sobre el índice de masa corporal (IMC) y otros parámetros antropométricos en niños con sobrepeso y obesidad en el periodo de un año.

\section{Pacientes y métodos}

Se realizó un estudio de comparación antes-después. La población de estudio incluyó a todos los pacientes de 2 a 14 años que acudieron a una consulta de Pediatría extrahospitalaria, durante el periodo de febrero de 2008 a marzo de 2011, que cumplían criterios de SP u OB, considerando sobrepeso cuando el IMC se encontraba entre los puntos de corte percentilares 85 y 95 y obesidad cuando era mayor o igual al percentil 95 según las curvas y tablas de crecimiento de la Fundación Orbegozo de $2004^{16}$.

Se consideraron criterios de exclusión la presencia de enfermedades genéticas, dismórficas o trastornos endocrinos diferentes al sobrepeso y la obesidad idiopática. Se calculó el tamaño de la muestra para un nivel de significación de 0,05 y una potencia del $80 \%$, asumiendo una disminución en el percentil del IMC de 8 unidades y una desviación típica de la variable diferencia de 25 unidades, ajustando para un $25 \%$ de pérdidas. Se obtuvo un tamaño muestral de 98 pacien- 
tes. Mediante muestreo consecutivo, se propuso participar en el programa "Aprendiendo a comer" a 162 niños, solicitando a los padres o tutores el consentimiento informado, 41 rehusaron participar. De los 121 pacientes incluidos, 20 abandonaron el programa tras la primera sesión y tres tras la quinta. La mayoría (85\%) por dificultades sociales o personales para implementar la dieta y una minoría (15\%) por miedo a inducir trastornos anoréxicos. El estudio fue aprobado por los Comités de Ética e Investigación del Hospital General de Ciudad Real.

\section{Intervenciones}

Hemos desarrollado el programa de educación nutricional "Aprendiendo a comer" para monitorizar los cambios hacia una alimentación correcta, debido a las dificultades para modificar hábitos de conducta enraizados en la familia. El programa consta de una visita mensual de unos 30 minutos por parte de la nutricionista y 15 minutos por parte del pediatra durante los cuatro primeros meses, y bimensual hasta completar el año de seguimiento del estudio. En la primera visita se explican los fundamentos de la $D M / T$, consistente en esencia en la dieta mediterránea que propone la Sociedad Española de Nutrición Comunitaria $(\text { SENC })^{14}$ y otras entidades científicas ${ }^{17-19}$. No hay diferencias en la proporción de nutrientes básicos entre la dieta de las guías tipo mediterránea ${ }^{14}$ (GTM) y la indicada en el estudio (DM/T). Únicamente al remarcar conceptos como frescos, crudos, perecederos, de temporada, integrales, con bajo índice/carga glucémica (IC/CG), abundantes en fibra y bajos en grasas, frente a los alimentos industriales, elaborados, atemporales, precocinados y con larga fecha de caducidad, conformarían un pequeño matiz diferencial que se resume en la tabla 1. Esto lo conseguimos en esencia, recomendando un consumo abundante de frutas, verduras, hortalizas, frutos secos, legumbres y cereales integrales. Acompañado de una moderación en el consumo de proteínas y grasas saturadas. Utilizando el aceite de oliva como principal grasa comestible y limitando la ingesta de harinas refinadas, patatas y azúcares.

El consejo dietético se realizó de forma individualizada a cada paciente y a sus padres, con la propuesta de que fuera seguido por toda la familia. No se desarrolló intervención específica programada sobre actividad deportiva ni sobre aspectos cognitivo-conductuales, salvo recomendaciones generales. En los controles mensuales se abordaron temas generales (los desayunos saludables, al- 


\begin{tabular}{|c|c|c|}
\hline & GTM & $\mathrm{DM} / \mathrm{T}$ \\
\hline Calorías & Sin restricción calórica & Sin restricción calórica \\
\hline IG/CG & No se tiene en cuenta & Bajo IG/CG \\
\hline Crudos & No se tiene en cuenta & Aumentar \\
\hline Fibra & Normal & Aumentar \\
\hline Cereales & Refinados e integrales & Integrales \\
\hline Azúcares & Con moderación & Evitar \\
\hline Patata & Limitar las patatas fritas & Evitar \\
\hline Bebidas azucaradas & Limitar el consumo & Evitar \\
\hline Leche y derivados & Entera: $2-3$ raciones/día & Desnatados y derivados \\
\hline Carnes y embutidos & Más de 3-4 veces/semana & $\begin{array}{l}\text { Menos de 3-4 veces/semana } \\
\text { Baja en grasas }\end{array}$ \\
\hline Aceite oliva & Uso preferente & Uso preferente de aceite virgen extra \\
\hline Precocinados & Sin abusar & Evitar \\
\hline Dieta para toda la familia & Sí & Sí \\
\hline
\end{tabular}

DM/T: dieta mediterránea/tradicional; GTM: guías tipo mediterránea; IG/CG: índice g/ucémico/carga glucémica.

muerzos y meriendas, frutas y verduras, masa magra, el área muscular del brazo cereales integrales) y temas específicos y el cociente cintura/cadera, tal y como relacionados con las mayores dificultades recomienda el comité de expertos de la ambientales (fiestas y cumpleaños, golo- Asociación Española de Pediatría ${ }^{17,18}$. El sinas y snacks, vacaciones, comer fuera peso y la talla se realizaron en ropa intede casa, comedor escolar, etc.).

Al inicio, en los controles intermedios y al acabar el programa, se determinaron las variables antropométricas -peso, taIla, IMC, pliegues cutáneos, perímetro del brazo, la cintura y la pelvis- y a partir de ahí se calcularon la masa grasa, la rior, con una báscula-tallímetro Seca ${ }^{\circledR}$ homologada. El IMC se calculó según la fórmula peso en kilogramos dividido entre el cuadrado de la talla en metros, y se tipificó a los pacientes según las curvas y tablas de la Fundación Orbegozo ${ }^{16}$. Los pliegues cutáneos se midieron con un pli- 
cómetro Holtain homologado y por un solo investigador, y se eligió el valor medio de tres mediciones consecutivas. Los perímetros de cintura y cadera y el perímetro del brazo fueron medidos por un único investigador tres veces consecutivas con una cinta métrica no metálica e inextensible; se tomó como valor el correspondiente a la media de las tres mediciones. El área muscular del brazo se calculó a partir de la medición de la circunferencia en el punto medio del brazo y de los valores del pliegue graso tricipita $^{20}$. El porcentaje de grasa corporal se calculó según las formulas de Slaugther et al. ${ }^{21}$.

Con el fin de evaluar los cambios introducidos y la calidad de la dieta mediterránea, hemos utilizado el test de KidMed $^{22-23}$, y en cada visita evaluamos y analizamos con los pacientes y sus padres las dificultades que fueron surgiendo y cómo podíamos modificar la conducta para obtener los mejores resultados.

\section{Análisis estadístico}

Se utilizó el paquete estadístico SPSS ${ }^{\circledR}$ 15.0. Se realizó un análisis descriptivo con estadísticos de tendencia central y de dispersión para variables cuantitativas y frecuencias absolutas y relativas para variables cualitativas. Las diferencias de los valores medios de las variables cuantitativas al inicio y al final de la intervención se analizaron mediante la prueba $t$ de Student para datos dependientes previa comprobación de la normalidad de la distribución de las variables mediante la prueba de Shapiro-Wilk, utilizando un intervalo de confianza del 95\% (IC 95\%). Para las variables categóricas se utilizó el test de MacNemar. Se fijó un nivel de significación $\alpha=0,05$.

\section{Resultados}

Participaron en el programa un total de 98 pacientes, de los cuales 55 eran varones y 43 mujeres. La edad media fue de 8,6 años (rango 2-14 años). El 53\% tenía OB y el $46 \%$ SP (tabla 2).

Tras un año de intervención (13,3 meses) el $61,2 \%$ de los pacientes se encontraba por debajo del percentil 85 del IMC, considerándose por lo tanto en normopeso. El 73,1\% dejó de ser obeso (IC 95\%: 56-78; $p<0,001$ ), y de los que tenían SP, el 82,6\% alcanzó valores normales (IC 95\%: 77-97; $p<0,001$ ) (figura 1). No se observaron diferencias significativas entre niños y niñas. El $96,6 \%$ acabó el estudio con un percentil del IMC más bajo que al inicio. En conjunto, el percentil del IMC descendió con la intervención desde un valor medio de $93,42 \pm 5,07$ a $79,5 \pm 14,41$, diferencia 


\begin{tabular}{|c|c|c|c|}
\hline & $\begin{array}{c}\text { Total } \\
\mathrm{N}=98\end{array}$ & $\begin{array}{c}\text { Niños } \\
N=55\end{array}$ & $\begin{array}{c}\text { Niñas } \\
\mathrm{N}=43\end{array}$ \\
\hline Edad en años* & $8,61(3,58)$ & $8,63(3,48)$ & $8,58(3,76)$ \\
\hline$I M C^{*}$ & $21,85(2,91)$ & $21,86(2,87)$ & $21,84(3,00)$ \\
\hline Percentil IMC & $93,42(5,07)$ & $94,09(4,75)$ & $92,56(5,39)$ \\
\hline Sobrepeso & $46(46,94 \%)$ & $26(47,3 \%)$ & $20(46,5 \%)$ \\
\hline Obesidad & $52(53,06 \%)$ & $29(52,7 \%)$ & $23(53,5 \%)$ \\
\hline \multicolumn{4}{|c|}{$\begin{array}{l}\text { IMC: índice de masa corporal. } \\
{ }^{*} \text { Media (desviación estándar). }\end{array}$} \\
\hline
\end{tabular}

media de 13,86 $\pm 1,54$ (IC 95\%: 10,8- Las mayores diferencias se obtuvieron 16,90; $p<0,001)$. Las variables antropo- en los cuatro primeros meses, con una métricas antes, a los cuatro meses y des- bajada del IMC de 1,16 que, reflejado en pués de la intervención se exponen en la percentiles del IMC, suponía una dismitabla 3.

Figura 1. Porcentaje de obesidad y sobrepeso al inicio y al final del estudio.
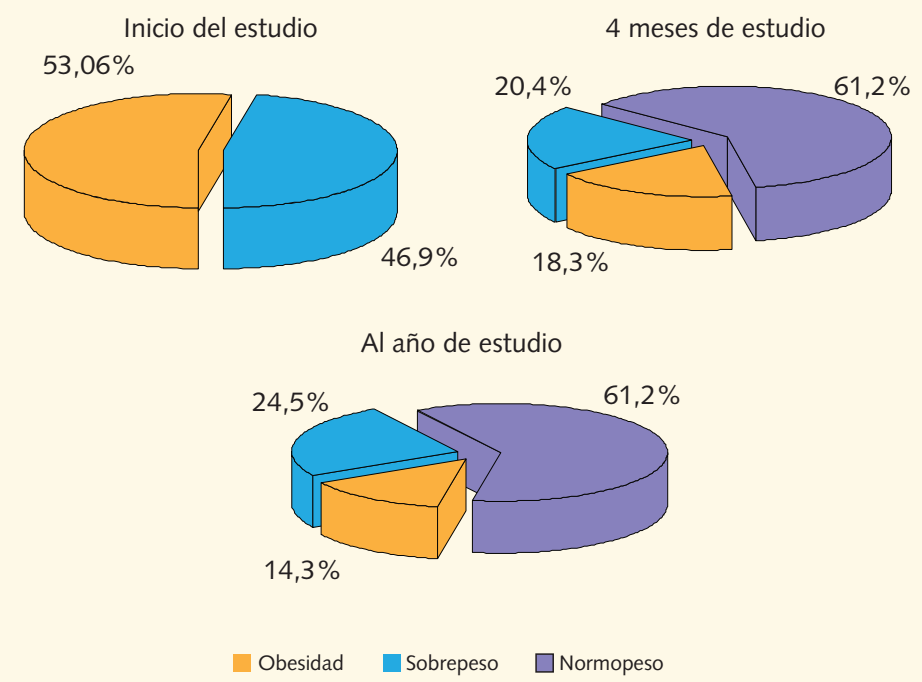


\begin{tabular}{|c|c|c|c|c|c|c|c|c|}
\hline & & \multirow{2}{*}{ Media } & \multirow{2}{*}{$\begin{array}{l}\text { Desviación } \\
\text { típica }\end{array}$} & \multirow{2}{*}{$\begin{array}{l}\text { Diferencias } \\
\text { entre el inicio } \\
\text { y el final del } \\
\text { tratamiento }\end{array}$} & \multirow{2}{*}{ IC (95\%) } & \multirow{2}{*}{$\mathbf{P}$} & \multicolumn{2}{|c|}{$\begin{array}{l}\text { Intervalo de confianza } \\
\text { para la media al } 95 \%\end{array}$} \\
\hline & & & & & & & $\begin{array}{l}\text { Límite } \\
\text { inferior }\end{array}$ & $\begin{array}{l}\text { Límite } \\
\text { superior }\end{array}$ \\
\hline \multirow{3}{*}{ Peso (kg) } & Inicio & 40,93 & 16,69 & \multirow{3}{*}{$2,41(2,36)$} & \multirow{3}{*}{$\begin{array}{l}7,09 a \\
2,25\end{array}$} & \multirow{3}{*}{0,342} & 37,58 & 44,27 \\
\hline & 4 meses & 40,01 & 16,08 & & & & 36,79 & 43,23 \\
\hline & Año & 43,34 & 16,47 & & & & 40,04 & 46,65 \\
\hline \multirow{3}{*}{ Altura (m) } & Inicio & 1,33 & 0,21 & \multirow{3}{*}{$0,7(0,3)$} & \multirow{3}{*}{$\begin{array}{l}0,13 a \\
0,01\end{array}$} & \multirow{3}{*}{0,063} & 1,28 & 1,37 \\
\hline & 4 meses & 1,35 & 0,21 & & & & 1,31 & 1,39 \\
\hline & Año & 1,40 & 0,20 & & & & 1,36 & 1,44 \\
\hline \multirow{3}{*}{ IMC (kg/m²) } & Inicio & 21,85 & 2,91 & \multirow{3}{*}{$0,8(0,4)$} & \multirow{3}{*}{$\begin{array}{l}-0,01 a \\
-1,62\end{array}$} & \multirow{3}{*}{0,017} & 21,27 & 22,44 \\
\hline & 4 meses & 20,69 & 2,87 & & & & 20,11 & 21,26 \\
\hline & Año & 21,05 & 2,91 & & & & 20,47 & 21,63 \\
\hline \multirow{3}{*}{$\begin{array}{l}\text { Percentil } \\
\text { de IMC }\end{array}$} & Inicio & 93,42 & 5,07 & \multirow{3}{*}{$13,86(1,54)$} & \multirow{3}{*}{$\begin{array}{l}-10,8 \mathrm{a} \\
16,9\end{array}$} & \multirow{3}{*}{0,0} & 92,40 & 94,44 \\
\hline & 4 meses & 80,46 & 14,39 & & & & 77,58 & 83,35 \\
\hline & Año & 79,56 & 14,41 & & & & 76,66 & 82,45 \\
\hline \multirow{3}{*}{$\begin{array}{l}\text { Pliegue } \\
\text { tricipital } \\
(\mathrm{mm})\end{array}$} & Inicio & 20,94 & 7,28 & \multirow{3}{*}{$3,02(0,91)$} & \multirow{3}{*}{$\begin{array}{l}-1,22 \mathrm{a} \\
-4,81\end{array}$} & \multirow{3}{*}{0,0} & 19,48 & 22,40 \\
\hline & 4 meses & 17,92 & 5,41 & & & & 15,45 & 17,62 \\
\hline & Año & 18,04 & 5,37 & & & & 15,86 & 17,99 \\
\hline Pliegue & Inicio & 17,90 & 8,30 & & & & 16,23 & 19,56 \\
\hline subescapular & 4 meses & 14,40 & 6,52 & $5,37(1,08)$ & $\begin{array}{l}-3,22 \mathrm{a} \\
-7,51\end{array}$ & 0,0 & 13,09 & 15,71 \\
\hline & Año & 12,53 & 6,85 & & & & 11,15 & 13,90 \\
\hline & Inicio & 68,47 & 10,00 & & & & 66,46 & 70,47 \\
\hline Cintura (cm) & 4 meses & 65,58 & 9,20 & $1,32(1,35)$ & $\begin{array}{l}-1,35 a \\
-4,00\end{array}$ & 0,100 & 63,73 & 67,42 \\
\hline & Año & 67,14 & 9,01 & & & & 65,34 & 68,95 \\
\hline & Inicio & 78,74 & 13,67 & & & & 76,00 & 81,48 \\
\hline Cadera $(\mathrm{cm})$ & 4 meses & 75,87 & 12,64 & $2,2(1,9)$ & $\begin{array}{l}6,01 \mathrm{a} \\
1,48\end{array}$ & 0,024 & 73,34 & 78,41 \\
\hline & Año & 81,01 & 12,95 & & & & 78,41 & 83,60 \\
\hline Índice & Inicio & 0,8767 & 0,073 & & & & 0,85 & 0,89 \\
\hline cintura- & 4 meses & 0,8702 & 0,061 & $0,041(0,01)$ & $\begin{array}{l}-0,01 \mathrm{a} \\
-0,06\end{array}$ & 0,0 & 0,85 & 0,88 \\
\hline cadera & Año & 0,8348 & 0,062 & & & & 0,82 & 0,84 \\
\hline & Inicio & 30,39 & 8,64 & & & & 28,65 & 32,11 \\
\hline $\begin{array}{l}\% \text { Masa } \\
\text { grasa }\end{array}$ & 4 meses & 26,38 & 6,90 & $5,66(1,14)$ & $\begin{array}{l}-3,41 \text { a } \\
-7,91\end{array}$ & 0,0 & 22,98 & 25,91 \\
\hline & Año & 24,71 & 7,28 & & & & 23,25 & 26,17 \\
\hline & Inicio & 27,43 & 9,22 & & & & 25,58 & 29,28 \\
\hline $\begin{array}{l}\% \text { Masa } \\
\text { magra }\end{array}$ & 4 meses & 29,44 & 10,04 & $4,46(1,43)$ & $\begin{array}{l}7,59 \text { a } \\
1,86\end{array}$ & 0,001 & 27,39 & 31,49 \\
\hline & Año & 31,90 & 10,85 & & & & 29,72 & 34,07 \\
\hline & Inicio & 6,587 & 1,92 & & & & 6,19 & 6,97 \\
\hline $\begin{array}{l}\text { RIDMED } \\
\text { (puntos) }\end{array}$ & 4 meses & 10,33 & 1,37 & $3,86(0,24)$ & $\begin{array}{l}3,35 a \\
4,29\end{array}$ & 0,0 & 10,06 & 10,61 \\
\hline & Año & 10,44 & 1,41 & & & & 10,16 & 10,73 \\
\hline
\end{tabular}

IC: intervalo de confianza; IMC: índice de masa corporal.

Prueba de comparación de medias: t de Student para datos apareados. 
los ocho meses restantes el percentil del IMC disminuyó ligeramente, pero no hubo diferencias significativas en el IMC, hecho en parte justificado por el aumento de edad y de envergadura (figura 2 ).

Los parámetros que evalúan la adiposidad periférica disminuyeron de forma significativa. En el conjunto de la población de estudio la pérdida de masa grasa fue del $18,7 \%$ respecto a los valores iniciales.

En los parámetros determinantes del crecimiento y desarrollo, como son la talla y la masa magra, se produjo un incremento adecuado, estadísticamente significativo.

El incremento de peso medio el año anterior al estudio fue de $5,7 \mathrm{~kg}$, casi el doble que los $2,4 \mathrm{~kg}$ actuales y el incremento de la talla media fue de $6,92 \mathrm{~cm}$ similar a los $7 \mathrm{~cm}$ actuales.
El área de masa magra del brazo aumentó adecuadamente, mientras que disminuyó significativamente el área de masa grasa. En la tabla 4 se recogen los resultados de los valores antropométricos según el sexo.

El $31 \%$ de los pacientes alcanzó valores de normalidad rápidamente en los cuatro primeros meses, el 34\% mejoró moderadamente y consolidó sus logros hasta el final, y el $35 \%$ bajó discretamente o mantuvo los niveles iniciales.

Los parámetros del test de KidMed mejoraron en el conjunto de la muestra al finalizar el programa. Se observó un aumento en el número de pacientes que consumían frutas, verduras, pescado, pasta, arroz y lácteos, así como en los que desayunaban cereales. Por otro lado, el porcentaje de pacientes que no desayunaban disminuyó, al igual que la pro-

Figura 2. Valores del percentil del índice de masa corporal y de la masa grasa al inicio y al final del estudio.

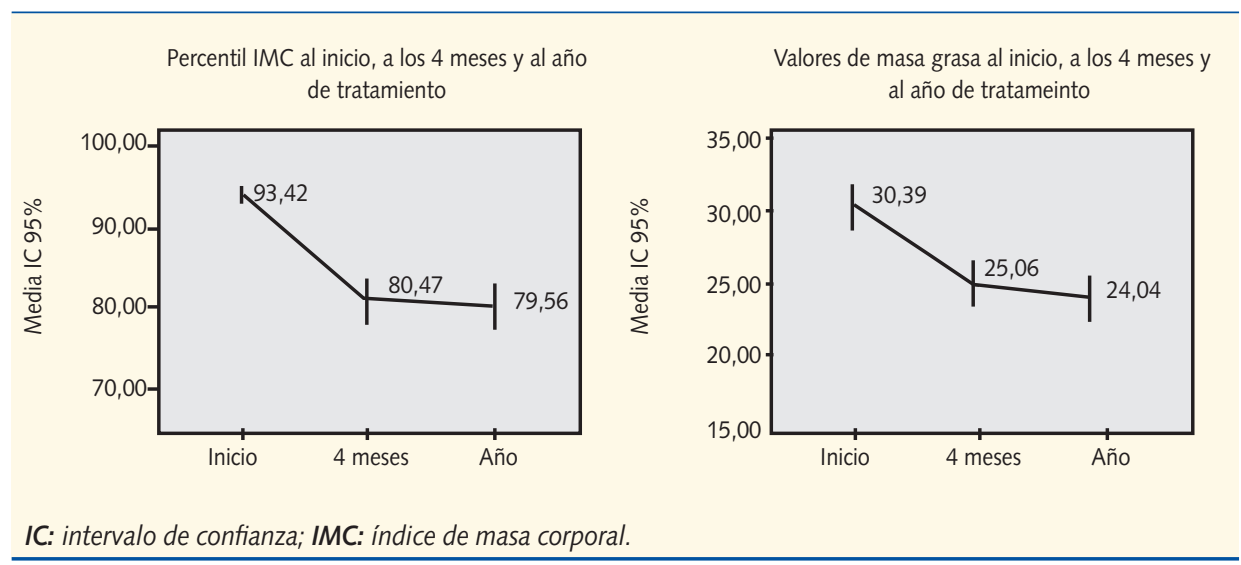




\begin{tabular}{|c|c|c|c|c|c|c|c|c|c|c|}
\hline & \multicolumn{5}{|c|}{ Niños (N = 55) } & \multicolumn{5}{|c|}{ Niñas $(\mathrm{N}=43)$} \\
\hline & Inicial & 4 meses & Final & DIF & $\mathbf{P}$ & Inicial & 4 meses & Final & DIF & $\mathbf{P}$ \\
\hline $\begin{array}{l}\text { Peso } \\
(\mathrm{kg})\end{array}$ & $\begin{array}{c}41,56 \\
(16,03)\end{array}$ & $\begin{array}{c}40,89 \\
(15,53)\end{array}$ & $\begin{array}{c}44,50 \\
(16,02)\end{array}$ & $\begin{array}{c}2,94 \\
(3,05)\end{array}$ & 0,44 & $\begin{array}{c}40,12 \\
(17,65)\end{array}$ & $\begin{array}{c}38,88 \\
(16,87)\end{array}$ & $\begin{array}{c}41,86 \\
(17,11)\end{array}$ & $\begin{array}{c}1,74 \\
(3,74)\end{array}$ & 0,64 \\
\hline Talla (m) & $\begin{array}{l}1,34 \\
(0,2) \\
\end{array}$ & $1,37(0,2)$ & $1,42(0,2)$ & $\begin{array}{c}0,07 \\
(0,03)\end{array}$ & 0,14 & $\begin{array}{c}1,31 \\
(0,23)\end{array}$ & $\begin{array}{c}1,33 \\
(0,22)\end{array}$ & $\begin{array}{c}1,37 \\
(0,21)\end{array}$ & $\begin{array}{c}0,06 \\
(0,04) \\
\end{array}$ & 0,19 \\
\hline IMC & $\begin{array}{l}21,86 \\
(2,87)\end{array}$ & $\begin{array}{l}20,75 \\
(2,86) \\
\end{array}$ & $\begin{array}{l}21,13 \\
(2,78) \\
\end{array}$ & $\begin{array}{l}-0,72 \\
(0,53)\end{array}$ & 0,11 & $\begin{array}{c}21,84 \\
(3,0)\end{array}$ & $\begin{array}{l}20,60 \\
(2,91)\end{array}$ & $\begin{array}{l}20,94 \\
(3,09)\end{array}$ & $\begin{array}{l}-0,89 \\
(0,65)\end{array}$ & 0,14 \\
\hline $\begin{array}{l}\text { Percentil } \\
\text { IMC }\end{array}$ & $\begin{array}{l}94,09 \\
(4,75)\end{array}$ & $\begin{array}{c}82,28 \\
(15,20)\end{array}$ & $\begin{array}{c}81,12 \\
(15,29)\end{array}$ & $\begin{array}{c}-12,96 \\
(2,16) \\
\end{array}$ & 0,001 & $\begin{array}{l}92,56 \\
(5,39)\end{array}$ & $\begin{array}{c}78,13 \\
(13,08)\end{array}$ & $\begin{array}{c}77,55 \\
(13,11)\end{array}$ & $\begin{array}{r}-15,01 \\
(2,16) \\
\end{array}$ & 0,001 \\
\hline $\begin{array}{l}\text { Masa } \\
\text { grasa } \\
(\%)\end{array}$ & $\begin{array}{l}29,48 \\
(8,20)\end{array}$ & $\begin{array}{l}25,84 \\
(6,68)\end{array}$ & $\begin{array}{l}24,09 \\
(7,39)\end{array}$ & $\begin{array}{l}-5,39 \\
(1,48)\end{array}$ & 0,001 & $\begin{array}{l}31,53 \\
(9,14)\end{array}$ & $\begin{array}{l}27,06 \\
(7,20)\end{array}$ & $\begin{array}{l}24,33 \\
(6,63)\end{array}$ & $\begin{array}{l}-7,20 \\
(1,72)\end{array}$ & 0,001 \\
\hline $\begin{array}{l}\text { Masa } \\
\text { magra } \\
(\%)\end{array}$ & $\begin{array}{l}28,33 \\
(9,11)\end{array}$ & $\begin{array}{l}29,64 \\
(9,81)\end{array}$ & $\begin{array}{c}33,15 \\
(10,99)\end{array}$ & $\begin{array}{c}4,81 \\
(1,92)\end{array}$ & 0,01 & $\begin{array}{l}26,28 \\
(9,33)\end{array}$ & $\begin{array}{c}27,55 \\
(10,34)\end{array}$ & $\begin{array}{c}30,91 \\
(11,02)\end{array}$ & $\begin{array}{c}4,63 \\
(2,20)\end{array}$ & 0,039 \\
\hline
\end{tabular}

DIF: diferencia; IMC: índice de masa corporal.

porción de los que consumían golosinas a diario y los que desayunaban bollería industrial (tabla 5).

El valor medio del índice KidMed al iniciar el programa era de 6,58 $\pm 1,92$ puntos (se interpreta como valor medio) y aumentó a 10,44 \pm 1,41 puntos (valor bueno-óptimo), diferencia media de $3,86 \pm 0,24$ (IC 95\%: 3,35-4,29; $p<$ $0,001)$. Los valores de adecuación al test de KidMed antes, al cuarto mes y al año del estudio se exponen en la figura 3.

\section{Discusión}

Nuestro programa de intervención se ha basado exclusivamente en la educación nutricional del niño y su familia. El núcleo de la intervención lo constituye el tipo de dieta utilizada, si bien los resultados obtenidos tenemos que relacionarlos con la intervención global, es decir, dieta en el contexto del programa de educación nutricional. El descenso en el percentil del IMC conlleva una disminución muy acentuada de la proporción de niños y niñas con SP y OB, en parte debido a que partimos de una población con un IMC medio no muy elevado y homogéneo, a diferencia de la población habitualmente atendida en las consultas especializadas. El descenso del IMC ha sido importante y es similar al comunicado en otros estudios con intervenciones a corto plazo $23-24$ y a otros donde, además de la intervención dietética, se realiza promoción de actividad física y se interviene 


\begin{tabular}{lccc}
\hline Tabla 5. Test de KidMed antes, a los cuatro meses y después de la intervención & \\
\hline & Inicio & 4 meses & Final \\
\hline Toma una fruta o zumo de fruta todos los días & $67 \%$ & $84 \%$ & $98 \%$ \\
\hline Toma una segunda fruta todos los días & $19 \%$ & $65 \%$ & $87 \%$ \\
\hline $\begin{array}{l}\text { Toma verduras frescas o cocinadas regularmente } \\
\text { una vez al día }\end{array}$ & $28 \%$ & $73 \%$ & $96 \%$ \\
\hline Toma verduras frescas o cocinadas más de una vez al día & $7 \%$ & $36 \%$ & $45 \%$ \\
\hline $\begin{array}{l}\text { Toma pescado fresco con regularidad (por lo menos dos } \\
\text { o tres veces por semana) }\end{array}$ & $65 \%$ & $83 \%$ & $98 \%$ \\
\hline Toma una vez o más a la semana comida de tipo fastfood & $13 \%$ & $2 \%$ & $2 \%$ \\
\hline Le gustan las legumbres & $80 \%$ & $85 \%$ & $98 \%$ \\
\hline Toma pasta o arroz casi a diario & $80 \%$ & $84 \%$ & $99 \%$ \\
\hline Desayuna un cereal o derivado & $70 \%$ & $84 \%$ & $97 \%$ \\
\hline Toma frutos secos con regularidad & $2 \%$ & $41 \%$ & $51 \%$ \\
\hline Utiliza aceite de oliva & $85 \%$ & $86 \%$ & $100 \%$ \\
\hline No desayuna & $12 \%$ & $1 \%$ & $0 \%$ \\
\hline Desayuna un lácteo & $79 \%$ & $85 \%$ & $97 \%$ \\
\hline Desayuna bollería industrial & $54 \%$ & $2 \%$ & $2 \%$ \\
\hline Toma dos yogures y/o queso diariamente & $73 \%$ & $84 \%$ & $99 \%$ \\
\hline Toma dulces y golosinas diariamente & $46 \%$ & $2 \%$ & $2 \%$ \\
\hline
\end{tabular}

sobre aspectos cognitivo-conductuales, precisando de recursos que complican su implementación en la práctica clínica ${ }^{25,26}$. En nuestra consulta, no disponemos de estos medios adicionales, que hubieran supuesto una mejoría en los resultados, aunque por otra parte nos ha permitido centrarnos exclusivamente en los aspectos dietéticos.

Una de las características fundamentales de la dieta utilizada es que no se aconseja restricción calórica. Las dietas hipocalóricas en el tratamiento de la $\mathrm{OB}$, con cálculo de calorías, conducen a una baja adherencia y a un menor impacto en la pérdida de peso que las dietas sin restricción calórica ${ }^{27}$. La DM/T es una dieta de bajo índice glucémico (IG) y baja carga glucémica (CG), por lo que hemos eliminado alimentos como los cereales refinados, el azúcar y la patata, que elevan excesivamente la insulinemia postprandial y originan alteraciones metabólicas no deseables. Cada día existen más evidencias sobre el papel que pueden jugar las denominadas dietas de bajo 
Figura 3. Calidad de la dieta, medida por el test de KidMed.

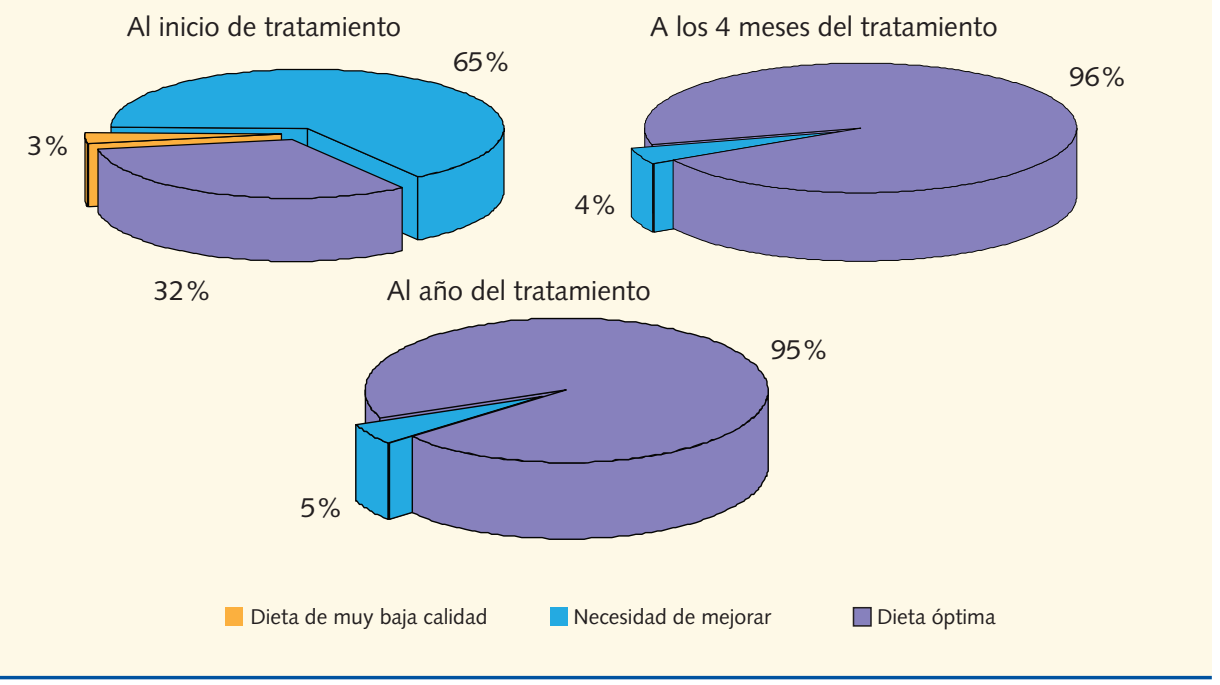

IG/CG en el tratamiento de las personas con sobrepeso y obesidad ${ }^{28-32}$. En un metaanálisis sobre dietas con bajo IG/CG en pacientes con obesidad ${ }^{33}$ se concluye que las personas obesas que realizan este tipo de dietas consiguen mayor pérdida de peso corporal que con dietas convencionales, sin necesidad de limitar la cantidad de alimentos ingeridos, resultando mejor aceptadas que las dietas más restrictivas. Además, las dietas de estas características incrementan la sensación de saciedad favoreciendo una menor ingesta calórica ${ }^{32-35}$. Aunque se han realizado algunos estudios en población infantil ${ }^{36,37}$, en general son escasos y de corto plazo ${ }^{38}$, por lo que hay que ser prudentes al generalizar estos resultados a la población pediátrica.

En general, este tipo de dietas basadas fundamentalmente en el IG/CG promovidas por la Escuela de Boston, se diferencian de la DM/T por el consumo elevado de proteínas y por la limitación de los carbohidratos ${ }^{32}$.

En el conjunto del año, los pacientes hicieron la mitad de peso que el año anterior, manteniendo el mismo crecimiento en altura, por lo que un porcentaje muy elevado $(96,6 \%)$ acabaron el estudio con un percentil del IMC más bajo que al inicio y el resto apenas subió de 
percentil. Esto puede sugerir que al menos desde un punto de vista preventivo, esta dieta podría ser eficaz para prevenir el SP y la OB.

Un tercio de los niños evolucionaron con una disminución del IMC muy acusada, alcanzando valores de normalidad rápidamente y manteniéndose en ella hasta el final del estudio. Otro tercio solo consiguió bajar ligeramente o mantener los percentiles del IMC, concordando con aquellos que peor cumplieron los criterios de la $\mathrm{DM} / \mathrm{T}$, en gran parte debido a circunstancias sociales y personales, ya que no excluimos del estudio a los niños que comían en el comedor escolar o fuera de casa, u otras circunstancias sociales que hacían difícil su cumplimentación. Por último, el tercio restante cumplimentó adecuadamente las normas de la $\mathrm{DM} / \mathrm{T}$, bajando el IMC moderadamente en los primeros cuatro meses, pero a partir de ahí solo consiguieron mantenerse en el percentil alcanzado, a pesar de que muchos de ellos hicieron todo lo posible por mejorarlo. Creemos que, al menos, estos muchachos y sus familias han adquirido unos conocimientos elementales de nutrición que les ayudarán a seguir defendiéndose adecuadamente de las dificultades del entorno. Nos faltaría poder complementar la dieta con el establecimiento de actividad deportiva, trata- miento cognitivo-conductual y educación social para la salud.

La dieta mediterránea ha sido contaminada por la actividad industrial, de modo que poco a poco nos vamos alejando de aquella alimentación basada en nuestros ancestros y que merece el apelativo de tradicional $(D M / T)^{39}$. Al eliminar las harinas refinadas, el azúcar y las patatas, y reducir la grasa saturada, eliminamos la mayoría de los alimentos industriales desnaturalizados. La piedra angular de nuestro programa educativo ha sido la de enseñar a los padres la diferencia entre alimentos frescos, crudos, perecederos y naturales, frente a la generalidad de los alimentos industriales.

El objetivo fundamental del tratamiento de la OB en la edad pediátrica es conseguir una reducción de la masa grasa, junto con la preservación del crecimiento y la masa magra ${ }^{21}$. La masa grasa disminuyó un 18,7\%, al tiempo que la talla y la masa magra se incrementaron adecuadamente. El programa nutricional trata de reforzar los conocimientos y habilidades de las familias y sus componentes, monitorizando la consecución de una dieta mediterránea de la máxima calidad, junto con la promoción de hábitos de conducta que favorezcan un mejor control de la alimentación y de la salud. La adherencia terapéutica, tanto del niño 
como de su familia, es un factor clave en el éxito de las intervenciones dietéticas.

Consideramos que la dieta que hemos utilizado, junto con el programa de educación dietética, es muy adecuada para el éxito de los objetivos. Es fácil de realizar en nuestro medio, con conocimientos y experiencia muy amplios por parte de la población, y va dirigida a todos los miembros de la familia sin excepción, dado que además de no tener restricción calórica es una dieta óptima en su contenido para todas las edades. Es destacable el alto grado de adecuación a la dieta mediterránea después de la intervención. Inicialmente, el test de KidMed daba valores considerados óptimos o buenos en el $32 \%$ de los pacientes, proporción ligeramente inferior que la obtenida en algunos estudios ${ }^{23,40}$, alcanzando al año una valoración óptima o buena en el 95\% de los niños que participaron en el programa, valores superiores a los obtenidos en otros estudios después de una intervención ${ }^{26}$. En el programa de seguimiento, vamos puntuando y estimulando su cumplimentación en cada una de las visitas, hasta conseguir una calificación óptima, que generalmente se ve correspondida con una mejoría en el control del peso.

En resumen, los resultados obtenidos son muy satisfactorios, mediante una intervención técnicamente sencilla, asequible desde Atención Primaria y con la posibilidad de llegar a prácticamente toda la población. Frente a las dietas hipocalóricas y de bajo índice glucémico, proponemos una dieta sin restricción calórica basada en la dieta mediterránea/tradicional. Consideramos que la dieta utilizada, si bien no podemos desligarla de la intervención en su conjunto, es el pilar fundamental de unos resultados prometedores.

\section{Agradecimientos}

A los doctores Ángel Nogales Espert y Alberto León Martín, por sus amables orientaciones y su disponibilidad para ayudarnos a mejorar este estudio.

\section{Bibliografía}

1. WHO. Overweight and obesity.[en línea] [actualizado en marzo de 2011; consultado el 29/11/2011]. Disponible en www.who.int/me diacentre/factsheets/fs311/en/

2. Serra L, Ribas L, Aranceta J, Pérez C, Saavedra $P$, Pena L. Obesidad en la infancia y la adoles- cencia en España. Resultados del estudio Enkid (1998-2000) Med Clin (Barc). 2003;121:725-32.

3. Dalmau J, Vitoria I. Prevención de la obesidad infantil: hábitos saludables. An Pediatr Contin. 2004;2(4):250-4.

4. Guo SS, Wu W, Chumlea WC, Roche AF. Predicting overweight and obesity in adulthood 
from body mass index values in childhood and adolescence. Am J Clin Nutr. 2002;76(3):653-8.

5. Bibbins-Domingo $\mathrm{K}$, Coxson P, Pletcher MJ, Lightwood J, Goldman L. Adolescent overweight and future adult coronary heart disease. N Engl J Med. 2007;357:2371-9.

6. Must $A$, Jacques PF, Dallal GE, Bajema CJ, Dietz WH. Long-term morbidity and mortality of overweight adolescents. A follow-up of the Harvard Growth Study of 1922 to 1935. N Engl J Med. 1992;327:1350-5.

7. Davison KK, Birch LL. Weight status, parent reaction, and self-concept in five-year-old girls. Pediatrics. 2001;107:46-53.

8. Cano A, Pérez I, Casares I. Obesidad infantil: opiniones y actitudes de los pediatras. Gac Sanit. 2008;22(2):98-104.

9. Calañas-Continente A, Arrizabalaga JJ, Caixás A, Cuatrecasas G, Díaz-Fernández J, García-Luna PP y cols. Guía para el manejo del sobrepeso y la obesidad en la preadolescencia y adolescencia. Endocrin Nutr. 2008;55(supl 4):60-77.

10. Grupo de trabajo de la guía sobre la prevención y el tratamiento de la obesidad infantojuvenil. Centro Cochrane Iberoamericano, coordinador. Guía de práctica clínica sobre la prevención y el tratamiento de la obesidad infantojuvenil. Madrid: Plan de Calidad para el Sistema Nacional de Salud del Ministerio de Sanidad y Política Social. Agencia d'Avaluació de Tecnología i Recerca Mèdiques; 2009. Guía de práctica clínica: AATRM N.․ 2007/25.

11. Summerbell CD, Ashton V, Campbell KJ, Edmnds L, Kelly S, Waters E. Intervenciones para tratar la obesidad infantil (Revisión Cochrane traducida). En: La biblioteca Cochrane Plus, 2007 Número 4, Oxford: Update Software Ltd. Disponible en http://update-software.com

12. Oude Luttikhuis $H$, Baur $L$, Jansen $H$, Shrewsbury VA, O'Maley C, Stolk RP et al. Intervenciones para el tratamiento de la obesidad en los niños. Cochrane Database of Systematic Reviews 2009, Issue 1. Art. No.:CD001872. DOI: 10. 1002/14651858.CD001872. pub2.

13. Bautista-Castaño I, Sangil-Monroy M, Serra-Majem L. Conocimientos y lagunas sobre la implicación de la nutrición y la actividad física en el desarrollo de la obesidad infantil y juvenil. Med Clin (Barc). 2004;123:782-93.

14. Dapcich V, Salvador G, Ribas L, Pérez C, Aranceta J, Serra $L$ y cols. Guía de la alimentación saludable. Madrid: Sociedad Española de Nutrición Comunitaria, Everest; 2005.

15. Buckland G, Bach A, Serra L. Eficacia de la dieta mediterránea en la prevención de la obesidad. Una revisión de la bibliografía. Rev Esp Obes. 2008;6(6):329-39.

16. Sobradillo B, Aguirre A, Aresti U, Bilbao A, Fernández-Ramos $C$, Lizárraga $A$ y cols. Curvas y tablas de crecimiento (Estudios Longitudinal y Transversal). Bilbao: Instituto de Investigación sobre Crecimiento y Desarrollo, Fundación Faustino Orbegozo Eizaguirre; 2004.

17. Lama RA, Alonso A, Gil-Campos M, Leis R, Martínez $\mathrm{V}$, Moráis A y cols. Obesidad infantil. Recomendaciones del comité de Nutrición de la AEP, (1. ${ }^{a}$ parte), Prevención. Detección precoz. Papel del pediatra. An Pediatr (Barc). 2006;65:60715.

18. Dalmau J, Alonso M, Gómez L, Martínez C, Sierra C. Obesidad infantil. Recomendaciones del Comité de Nutrición de la Asociación Española de Pediatría. Parte II. Diagnostico. Comorbilidades. Tratamiento. An Pediatr (Barc). 2007;66:294-304.

19. Toledano E, Aznar S, Cortés O, Ferreira I, Gandarillas AM, Grima A y cols. 1. ${ }^{a}$ Conferencia de Prevención y Promoción de la Salud en la Práctica Clínica en España. Prevención de la Obesidad infantil y juvenil. Madrid: Ministerio de Sanidad y Consumo; 2009.

20. Frisancho AR. Antropometric standards for the assessment of growth and nutritional status. 
Ann Arbor: The University of Michigan Press; 1993.

21. Slaugther M, Lohman $T$, Boileau R, Horswill C, Stillman R, Val Loan M et al. Skinfold equations for estimation of body fatness in children and youths. Hum Biol. 1988;60:709-23.

22. Serra L, Ribas L, Ngo J, Ortega RM, Pérez $C$, Aranceta J. Alimentación, jóvenes y dieta mediterránea en España. Desarrollo del KidMed, índice de calidad de la dieta mediterránea en la infancia y la adolescencia. En: Serra L, Aranceta J (eds.). Alimentación infantil y juvenil. Estudio enKid. Barcelona: Masson; 2002.

23. Serra L, Ribas L, Aranceta J, Pérez C, Saavedra P, Peña L. Obesidad infantil y juvenil en España. Resultados del estudio EnKid (1998-2000). Med Clin (Barc). 2003;121:725-32.

24. Velázquez L, Rico J, Torres $M$, Medina $P$, Toca L, Escobedo J. Impacto de la educación nutricional sobre alteraciones metabólicas en niños y adolescentes con obesidad. Endocrinol Nutr. 2009;56(10):441-6.

25. García-Reyna N, Gussinyer S, Carrascosa A. Niñ@s en movimiento, un programa para el tratamiento de la obesidad infantil. Med Clin (Barc). 2007;129:619-23.

26. Gussinyer S, García-Reyna N, Carrascosa A, Gussynier M, Yeste D, Clemente $M$ y cols. Cambios antropométricos, dietéticos y psicológicos tras la aplicación del programa "Niñ@s en movimiento" en la obesidad infantil. Med Clin (Barc). 2008;131:245-9.

27. Fontana C, Damonte C, Pregliasco P, Roggi C. Hypocaloric diet and normocaloric diet in outpatient treatment in a group of children and adolescents. Pediar Med Chir. 2007;29:336-9.

28. Jenkins $D$, Wolever $T$, Taylor $R$, Barrer $H$, Hieden $\mathrm{H}$, Baldwin J. Glycolic index of foods: a phisiological basis for carbohydrates. Am J Clin Nutr. 1981;34:362-6.
29. Brand J, Holt S, Pawlak D, McMillan J. Glycemic index and obesity. Am J Clin Nutr. 2002;76(suppl):281S-5S.

30. Méndez M, Covas MI, Marrugat J, Vila J, Schröder H. Glycemic load, glycemic index, and body mass index in Spanish adults. Am J Clin Nutr. 2009;89:316-22.

31. Foster-Powell K, Holt S, Brand-Miller J. International tables of glycemic index and glycemic loads values. Am J Clin Nutr. 2002;76:5-56.

32. Ludwing D. Dietary glycemic index and obesity. J Nutr. 2000;130(2S Suppl):S280-S283).

33. Thomas DE, Elliot EJ, Baur L. Dietas de bajo índice glucémico o baja carga glucémica para el sobrepeso y la obesidad (Revisión Cochrane traducida). En: La biblioteca Cochrane Plus, 2007 Número 4. Oxford: Update Software Ltd. Disponible en www.update-software.com

34. Ball SD, Keller KR, Moyer-Mileur LJ, Ding YW, Donaldson D, Jackson WD. Prolongation of satiety after low versus moderately high glycemic index meals in obese adolescents. Pediatrics. 2003;111:488-94.

35. Ludwing D. The glycemic index. Physiological mechanisms relating to obesity, diabetes and cardiovascular disease. JAMA. 2002;287:241423.

36. Ebbeling $C$, Leidig $M$, Sinclair $K$, SegerShippee $L$, Feldman $H$, Ludwing D. Effects of an ad libitum low-glycemic load diet on cardiovascular disease risk factors in obese young adults. Am J Clin Nutr. 2005;81:976-82.

37. Ebbeling $C$, Leidig $M$, Feldman $H$, Lovesky $M$, Ludwing D. Effects of a low-glycemic load vs low-fat diet in obese young adults. JAMA. 2007;297:2092-102.

38. Spieth LE, Harnish HD, Lenders CM, Raezer LB, Pereira MA, Hangen SJ et al. A low-glycemic index diet in the treatment of pediatric obesity. Arch Pediatr Adolesc Med. 2000;154:947-51. 
39. Varela G, Ávila J, Cuadrado C, Del Pozo S, Ruiz E, Moreiras O. Valoración de la Dieta Española de acuerdo al Panel de Consumo Alimentario. Madrid: Ministerio de Medio Ambiente y Medio Rural y Marino. Secretaría General Técnica; 2008.
40. Mariscal-Arcos M, Rivas A, Velasco J, Ortega $M$, Caballero AM, Olea-Serrano F. Evaluation of the Mediterranean Diet Quality Index (KIDMED) in children and adolescents in Southern Spain. Public Health Nutr. 2008;12:1408-12. 
